\title{
Nouvelles expressions judéo-maghrébines, dossier coordonné par Guy Dugas et Sonia Zlitni Fitouri
}

\section{Carminella Biondi}

\section{(2) OpenEdition}

1 Journals

\section{Edizione digitale}

URL: http://journals.openedition.org/studifrancesi/1490

DOI: $10.4000 /$ studifrancesi. 1490

ISSN: 2421-5856

\section{Editore}

Rosenberg \& Sellier

\section{Edizione cartacea}

Data di pubblicazione: 1 décembre 2015

Paginazione: 641-642

ISSN: 0039-2944

\section{Notizia bibliografica digitale}

Carminella Biondi, «Nouvelles expressions judéo-maghrébines, dossier coordonné par Guy Dugas et Sonia Zlitni Fitouri», Studi Francesi [Online], 177 (LIX | III) | 2015, online dal 01 décembre 2015, consultato il 11 janvier 2021. URL: http://journals.openedition.org/studifrancesi/1490 ; DOI: https:// doi.org/10.4000/studifrancesi. 1490

Questo documento è stato generato automaticamente il 11 janvier 2021.

\section{(c) $($ ) $\odot$}

Studi Francesi è distribuita con Licenza Creative Commons Attribuzione - Non commerciale - Non opere derivate 4.0 Internazionale. 


\title{
Nouvelles expressions judéo- maghrébines, dossier coordonné par Guy Dugas et Sonia Zlitni Fitouri
}

\author{
Carminella Biondi
}

\section{NOTIZIA}

«Expressions maghrébines», vol. 13, n. 2, hiver 2014, Nouvelles expressions judéomaghrébines, dossier coordonné par Guy DUGAS et Sonia zLITNI FITOURI, pp. 187.

1 Il dossier si apre con un omaggio di Denise BRAHIMI à Guy Dugas, giustamente considerato come il padre degli studi sulla letteratura giudaico-maghrebina che, a partire dagli anni'90 del secolo scorso, data di pubblicazione della sua tesi sull'argomento, non ha mai smesso di battersi perché ne fosse riconosciuta la specificità all'interno della letteratura maghrebina di lingua francese. Una battaglia vittoriosa, come conferma anche questo importante dossier, che si apre con un'introduzione della co-curatrice Sonia zLITNI FITOURI, dal titolo Expressions judéomaghrébines: "Une force qui demeure" (pp.5-11) e si chiude con un aggiornamento bibliografico a cura di Guy DUGAS: Trente ans de littérature judéo-maghébine (1982-2013) (pp. 53-171), che, con i suoi quasi cinquecento titoli, ne indica la vitalità, malgrado la difficile situazione attuale degli ebrei in quei territori musulmani in cui hanno vissuto in pace da secoli.

2 Sonia Zlitni Fitouri apre la sua introduzione cercando di riassumere gli elementi che costituiscono la specificità della letteratura giudaico-maghrebina e che giustificano, almeno in parte, le scansioni del dossier, che indicheremo in seguito: «Marquée des thèmes constants tels que l'Histoire, l'exil, l'identité, la mémoire, la nostalgie, la littérature judéo-maghrébine de langue française aspire désormais à une forme de pluralisme qui «raconterait les mille et une façons d'être juif que les écrivains et les artistes vont inscrire dans leurs œuvres; les mille et une façons de vivre la judéité, de la 
rejeter, de la vivre obliquement, de la tenir à distance, de la mimer, de la stimuler, de la représenter, de la déconstruire, de la réinventer, de l'imaginer. Pas plus d'identité juive stabilisée que les autres, mais une mouvance, une fluidité». La citazione, tratta dal saggio di Régine Robin, Le Golem de l'écriture. De l'autofiction au cyber soi (Montréal, XYZ, 1997, p. 29), sottolinea la ricchezza di una scrittura che si è liberata degli schemi fissisti di un'identità ebraica monolitica, per modellarsi sulla complessità di un vissuto che ha comportato e comporta traumi, diaspore, contaminazioni. Alle caratteristiche indicate da Zlitni Fitouri, che nel complesso non sembrano poi davvero molto diverse da quelle che si potrebbero usare per definire la letteratura maghrebina di lingua francese, e forse tante altre letterature contemporanee, Guy DUGAS, nel suo articolo di chiusura (Fantaisie, littérature mémorieuse, iconophilie... Retour sur quelques concepts discutables, pp.139-152), ne aggiunge una che sembra meglio connotare questa letteratura: la dimension fantaisiste, la dimensione ludica, il gusto del paradosso, dell'humour, della parodia, surrogata da un'iconofilia che, mentre sembra ancorare al reale, all'oggettività, permette in realtà infiniti giochi della memoria e della scrittura.

Dicevo che gli elementi individuati come caratterizzanti di questo tipo di letteratura, spiegano anche in larga misura la scansione del dossier, che si divide in quattro parti: «Négocier une nouvelle identité»; «Marcher sur l'oubli»; «Supports visuels, supports mémoriels»; "Conceptualisation». I due articoli che compognono la prima parte si concentrano su due autori che rappresentano molto bene la ricerca e, allo stesso tempo, la difficoltà di una ricollocazione identitaria da parte di personaggi che non trovano più un loro contesto di riferimento o che cercano di riadattarlo al loro processo di trasformazione. Si tratta dei saggi di Debbie BERNARD, Ironie, altérité et racisme dans "Le café de Madame Ben Djamil" de Claude Kayat (pp.15-27) e di Mohamed ChaGRAOUI, Tunisianité, judéité et égalité dans les écrits de Gilbert Naccache (pp. 29-44). La seconda parte, composta, come la prima, di due articoli, si focalizza sul tema della memoria e della sua perdita (dovuta ai processi di assimilazione) e sul suo rapporto con la storia: Wafa BEN AZIZA, Nouvelles expressions judéo-maghrébines entre mémoire et histoire: une écriture en évolution (pp.47-60); Danielle DAHAN-FEUCHT, Marlène Amara: du silence à l'expression revendiquée d'une mémoire (pp.61-75). La terza parte si compone di tre articoli che non analizzano testi scritti, ma fenomeni visivi, che vanno dalla permanenza di una piccola sinagoga alla periferia di Tunisi, all'iconografia utilizzata sulle pagine di copertina nei testi giudaico-maghrebini, passando per l'analisi di un film che racconta l'esperienza negativa di ebrei marocchini trasferitisi, negli anni Sessanta, in Israele, dove non si riconoscono e non sono riconosciuti: Dora CARPENTER-LATIRI, Visites de la sinagogue de La Goulette. La synagogue Beith Mordechai, rue Khaznadar, témoin et miroir d'une minorité de Tunisie (pp. 79-98); Denise BRAHIMI, À propos des Judéo-berbères marocains, un film: "Tinghir-Jérusalem" (pp. 99-103); EWa TARTAKOWSKY, Littérature des auteurs d'origine judéo-maghrébine. Les couvertures, masques à visage découvert (pp. 105-121). L'ultima parte, sulla concettualizzazione, contiene il già citato articolo di Guy DUGAS, che cerca di enucleare uno specifico giudaico-maghrebino, pur nelle infinite varianti e trasformazioni di questa letteratura, specifico che individua, come si è detto, nell'aspetto ludico, nella sua volontà di sovversione e nel suo amore per l'iconofilia che alimenta la memoria. Mentre il saggio di Zakharia FATIH, The Aesthetics of Fragmentation, or a Way to Read El Maleh (pp.125-137), propone un nuovo approccio critico all'opera di El Maleh, modellato sul procedimento frammentario della sua scrittura. 
4 Un dossier importante, che cerca di fare il punto sulla scrittura giudaico-maghrebina, sia pure in maniera non sistematica, ma attraverso una serie di indagini su autori, opere o orientamenti culturali rappresentativi che ci permettono, comunque, una visione abbastanza esaustiva di questo fenomeno letterario. La bibliografia delle opere dell'ultimo trentennio che chiude il dossier e completa le bibliografie dei periodi precedenti, curate anch'esse da Guy Dugas, è quanto mai preziosa per qualsiasi tipo di ricerca si intenda intraprendere su questa letteratura. 